\section{Visión Electrónica \\ Más que un estado sólido}

https://revistas.udistrital.edu.co/index.php/visele

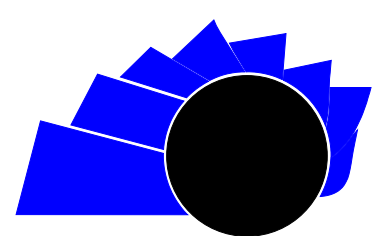

VISIÓN ELECTRÓNICA

VISIÓN INVESTIGADORA

\title{
Dispositivo háptico vibrotáctil inalámbrico para asistencia de actividades motoras
}

\author{
Wearable Haptic Vibrotactile System for Assistance of Motor Activities \\ Jonathan Roberto Torres Castillo. ${ }^{1}$, Juan Salvador Pérez Lomeli. ${ }^{2}$, Esperanza Camargo Casallas. $^{3}$, \\ Miguel Ángel Padilla Castañeda. ${ }^{4}$,
}

\section{INFORMACIÓN DEL ARTÍCULO}

Historia del artículo:

Enviado: 26/10/2017

Recibido: $11 / 11 / 2017$

Aceptado: $12 / 01 / 2018$

Palabras clave:

Déficit de movimiento

Percepción táctil

Retroalimentación háptica.

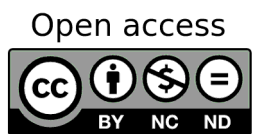

Keywords:

Deficit of movement

Touch perception

Haptic feedback.

\section{RESUMEN}

El presente artículo describe la investigación que condujo al desarrollo de un dispositivo háptico inalámbrico, capaz de generar estímulos mecánicos vibrotáctiles en diferentes puntos de la piel —y a frecuencias deseadas - por medio de dieciséis actuadores contenidos en un brazalete portátil diseñado para cualquier extremidad del cuerpo humano. Este prototipo permite tener control sobre cada actuador usado como punto de estimulación, accionado de forma independiente por medio de comandos transmitidos inalámbricamente hacia un sistema de control autónomo recargable dispuesto en el brazalete. Se realizaron pruebas de usabilidad, con respecto a la percepción táctil, que comprobaron el correcto funcionamiento del dispositivo. En perspectiva, el desarrollo, luego de una variedad de pruebas de validación con una amplia muestra de pacientes con y sin neuropatías, tiene como fin la creación de una base de datos para ser usada como valores consigna frente a estos pacientes — esperando que el sistema se use también en pacientes con déficit de movimiento-, y empleando la percepción táctil como estimulante psicomotor en la ejecución de actividades motoras.

\section{ABSTRACT}

This article describes the research that leads to the development of a wireless haptic device capable of generating vibrotractile mechanical stimuli at different points in the human skin and at desired frequencies by means of 16 actuators contained in portable bracelet isolated, designed for any extremity of the human. This prototype allows control over each actuator used as a test point, independently driven by commands transmitted wirelessly to a self- contained rechargeable control system placed on the bracelet. The completion and use of this system allowed to perform usability tests with respect to tactile perception from three experiments in healthy people, who verified the efficiency of the vibrotactile flux in the discrimination of nearby stimuli and the correct functioning of the device. With the development of this device want to perform it a wide variety of validation tests with a large sample of patients with and without neuropathies, in order to create a database to be used as set values against these patients and it is expected that the system is also used in patients with movement deficit, using tactile perception as a psychomotor stimulant in the execution of motor activities.

\footnotetext{
${ }^{1}$ Ingeniero en telecomunicaciones, Universidad Distrital Francisco José de Caldas. Magíster en Ingeniería Eléctrica, Universidad Nacional Autónoma de México. Correo electrónico: jonathanrtc@comunidad.unam.mx

${ }^{2}$ Diseñador industrial, Universidad Autónoma de Guadalajara. Técnico académico, Universidad Nacional Autónoma de México. Correo electrónico: salvador. perez@ccadet.unam.mx

${ }^{3}$ Ingeniera en control electrónico e instrumentación, Universidad Distrital Francisco José de Caldas. Doctora en Ingeniería, Pontificia Universidad Javeriana. Correo electrónico: especamargo@hotmail.com

${ }^{4}$ Ingeniero en computación, Universidad Nacional Autónoma de México. Doctor en Tecnologías de la Innovación y Robótica, Escuela Superior Sant 'Anna de Pisa. Correo electrónico: miguel.padilla@ccadet.unam.mx 


\section{Introducción}

Una discapacidad motriz en una persona puede ser causada por diferentes razones, principalmente por accidentes de diferente tipo o por enfermedades o lesiones neuronales que, de una $\mathrm{u}$ otra forma, paralizan de manera parcial o total el movimiento de las extremidades superiores e inferiores del cuerpo humano. Al surgir este tipo de discapacidad, es necesario iniciar una fase de rehabilitación - que requiere diferentes etapas y metodologías - con tiempos de asistencia prolongados, por lo que los pacientes tienden a abandonar los procedimientos dejando inconcluso el tratamiento de recuperación.

Adicionalmente, existe un continuo crecimiento a nivel mundial de pacientes con necesidades de terapias de rehabilitación, lo que ha incrementado la presión sobre los sistemas de salud y, consecuentemente, la reducción de los tiempos y recursos disponibles para los tratamientos.

Con respecto a la rehabilitación, es a menudo un proceso tedioso y largo que, incluso, puede prolongarse por meses, aun si se realizan varias sesiones a la semana; esta se lleva a cabo mediante visitas recurrentes a centros de rehabilitación y fisioterapia en casa, involucrando valiosos recursos humanos [1]. Bajo estas consideraciones, es esencial investigar formas más efectivas de realizar las terapias, por lo que seguir un enfoque tecnológico es muy prometedor.

En el anterior sentido, actualmente la tecnología y la robótica ofrecen a los usuarios multitud de dispositivos avanzados orientados a la investigación, a la ciencia, a la industria o al entretenimiento, capaces de simular con gran realismo distintas sensaciones hápticas (propioceptivas, táctiles y vestibulares). Dichos dispositivos se caracterizan por proporcionar contacto físico entre el ordenador y el usuario, así como realimentación de fuerza y táctil al sujeto que interactúa con entornos virtuales o remotos $[2,3]$. Estos dispositivos avanzados son capaces de hacer creer al usuario que tocan o colisionan con objetos virtuales, sólidos o deformables, también son capaces de proporcionar diferentes texturas hápticas dependientes del tipo de superficie del objeto (rugosa, lisa, sin fricción, pegajosa, penetrable, entre otras).

Por otra parte, la investigación sobre el uso de tecnologías robóticas y de realidad virtual en pacientes con déficits neuromotores se ha extendido en los últimos años $[4,5]$. A pesar de que los resultados reportados en la literatura parecen prometedores, y sus posibles beneficios extenderse a diversas poblaciones de pacientes, su aplicación está fuertemente restringida a hospitales regionales a gran escala, pero muy limitada para clínicas locales de fisioterapia, además de estar prácticamente no disponibles para programas de entrenamiento en casa [6].

Lo anterior ha motivado el reciente crecimiento de la realidad virtual aplicada a videojuegos serios para rehabilitación; tales juegos tienen un impacto potencialmente alto debido a su fácil distribución, su aplicabilidad en un rango más amplio de discapacidades motoras y su potencial para asignar protocolos de entrenamiento en "altas dosis" y "alta intensidad" $[7,8]$. Sin embargo, a pesar de las extraordinarias mejoras tecnológicas en este campo, aún falta mucho por investigar. Entre las problemáticas por abordar se tienen: (a) la ausencia o limitada posibilidad de retroalimentación sensomotora en forma de contacto táctil; (b) limitados esquemas de interacción, principalmente orientados a tareas muy genéricas que involucran alcanzar y acertar objetos virtuales móviles, los cuales están aún lejos de las situaciones de la vida cotidiana real de los pacientes; (c) limitado realismo, en general enfocado solo a aspectos visuales, pero sin convincente realismo físico, lo que limita la posibilidad de asignar tareas motoras funcionales progresivamente más complejas y que requieran mayores destrezas finas; (d) muchos sistemas son genéricos, pero no enfocados a déficits específicos; (e) un pobre entendimiento de papel que desempeña en la recuperación el asignar diferentes niveles de dificultad de la terapia y sobre todo de cómo asignar la dosis óptima, lo anterior de acuerdo con las condiciones clínicas y personales de los pacientes; (f) la reducida validación y evaluación clínica extensiva en estudios controlados de muchos sistemas; (g) la necesidad de entender la relación entre las características de este tipo de sistemas y su impacto en la recuperación de los pacientes.

Actualmente también es factible el uso de tecnologías de realidad virtual y cómputo móvil en sistemas de rehabilitación, asequibles en ámbitos locales o caseros; sin embargo, tales sistemas tienen la importante limitación de carecer de capacidades de actuación y control motor al no estar integrados con algún sistema robótico. Con el objetivo de proporcionar información al paciente sobre su desempeño/errores, sería benéfica la retroalimentación háptica en ese tipo de sistemas de realidad virtual durante el entrenamiento, a través de la introducción del sentido del tacto en el ciclo de retroalimentación multisensorial y aumentando los canales visuales y auditivos [9]. Más aún, tener la sensación táctil de 
tocar objetos virtuales — además del audio y la visiónpodría jugar un papel preponderante para ayudar en el proceso de reaprendizaje de habilidades manuales de control de los movimientos en los pacientes; por lo tanto, es importante la integración de dispositivos portátiles capaces de proveer retroalimentación háptica, aunque esto ha recibido poca atención por la comunidad científica - dando como resultado una literatura precaria - , pero poco a poco ha comenzado a despertar cada vez más interés.

El documento se estructura de la siguiente manera: en primer lugar se establecen los materiales y métodos para el desarrollo del sistema de retroalimentación háptico vibrotáctil inalámbrico (DRHVI); luego se describe detalladamente el DRHVI propuesto; posteriormente, se exhiben las pruebas de usabilidad del dispositivo en tres tipos de experimentos con una persona sana sin discapacidad motriz ni déficit sensorial; luego se muestran y discuten los resultados de la validación del DRHVI y, finalmente, se muestran las conclusiones de la investigación.

\section{Metodología y materiales}

De acuerdo con la motivación expuesta, se desarrolló un DRHVI que al usarse junto a sistemas complementarios sirva como base para investigaciones futuras en el área de asistencia en la ejecución de actividades motoras de rehabilitación. El prototipo de DRHVI está compuesto por tres sistemas: mecánico, electrónico e interfaz de usuario. Para su diseño y desarrollo se establecieron seis fases: (a) caracterización de los actuadores; (b) elección de dispositivo electrónico de control; (c) elección del tipo de comunicación; (d) diseño de sistema de alimentación; (e) integración del sistema para realizar validación sensorial; (f) validación de flujo vibrotáctil.

Estableciéndose como resultado que el sistema de retroalimentación háptico conste de dieciséis actuadores vibrotáctiles (minivibromotores), los cuales pueden ser accionados de forma aleatoria o precisa; también controlando el número de motores a operar y sus diferentes frecuencias de forma independiente.

El control se realiza de manera remota con un sistema que establece la comunicación a través de dos módulos Xbee, donde un módulo funciona como transmisor (Tx) conectado al equipo central de control, dotado por una interfaz gráfica de usuario capaz de enviar comandos inalámbricamente al otro módulo receptor
$(\mathrm{Rx})$, conectado a su vez con un sistema de control que interpreta dicho comando para generar señales PWM a los diferentes actuadores, regulando los niveles de vibración a través del aumento o la disminución del ancho de pulso. Posteriormente, se acoplan todos los elementos que conforman el sistema (actuadores, dispositivos de comunicación, control y alimentación) en un brazalete portátil.

Como método de validación del DRHVI se optó por aplicar pruebas de usabilidad psicofísicas y sensoriales.

\section{Desarrollo del sistema de retroalimentación háptico vibrotáctil inalámbrico}

Para el propósito de la investigación se estableció un perfil de uso en hombres y mujeres entre 16 y 65 años (edad de productividad laboral) pertenecientes a cualquier estrato socioeconómico, con alguna discapacidad neuromotora (accidente cerebro-vascular) o musculoesqueléticas (reumatismo), o bien con déficit sensorial por neuropatía (diabéticos), pueden presentar una condición de discapacidad transitoria que afecte su calidad de vida debido a la imposibilidad de realizar las actividades de la vida diaria con normalidad. Esta información sugirió la definición de variables de tamaño, forma y frecuencia necesarias para el diseño de la estructura de soporte en la interfaz del dispositivo, lo anterior con el fin de que sea cómodo para el paciente.

\subsection{Características de diseño del dispositivo}

Para controlar el comportamiento de cada uno de los actuadores se diseñó el dispositivo de comunicación y control inalámbrico (Figura 1), en este se implementó como base un microcontrolador Arduino NANO que permite accionar los dieciséis actuadores por medio de señales PWM de manera simultánea, al mismo tiempo que transmite y recibe comandos de control de forma inalámbrica a través de un módulo de comunicación Xbee.

La función del Arduino Nano es la de interpretar el comando recibido desde el Xbee, receptor que funciona bajo las especificaciones 802.15.4. Este comando contiene la información del motor que se debe activar, especificando número de motor, frecuencia de vibración y el tiempo que debe ser accionado. El módulo permite la transmisión de datos punto a punto, o punto a multipunto con una velocidad de hasta $250 \mathrm{Kbits} / \mathrm{s}$ y opera en la banda de frecuencias ISM (Industrial, Scientific and Medica). 
Figura 1: Diseño esquemático del acople entre el sistema de control y el sistema de comunicación hecho con Proteus 8 .

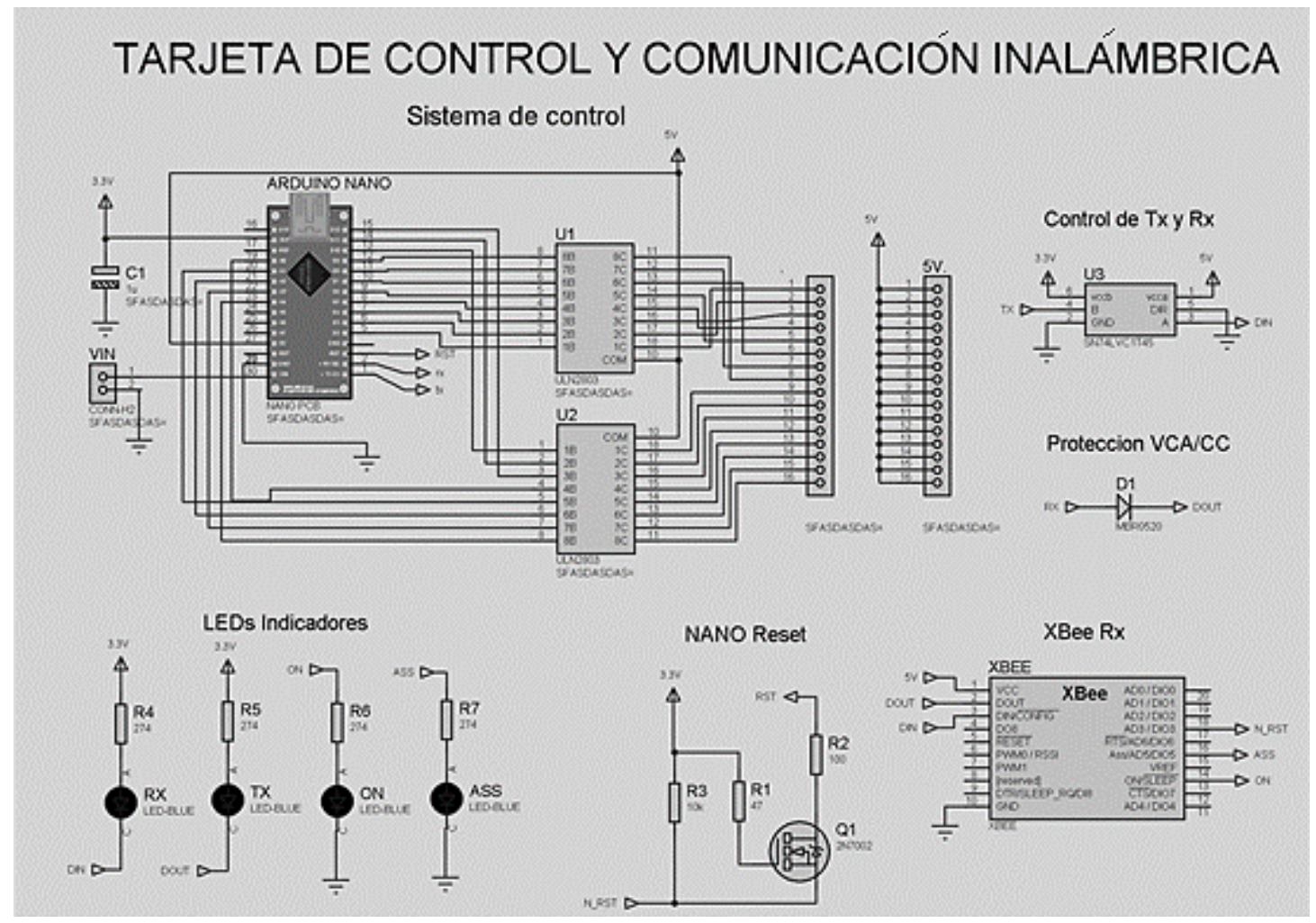

Fuente: elaboración propia.

Como se utilizaron dos módulos Xbee, uno conectado a la interfaz ( $\mathrm{Rx}$ ) y otro conectado al ordenador ( $\mathrm{Tx})$, se logró interactuar de manera remota con los actuadores que generan sensaciones hápticas.

Luego se obtuvo la tarjeta electrónica doble capa con los pads y superficies metalizadas en las cuales se montaron los componentes (Figura 2). Esta interfaz tiene un tamaño menor al esperado, con un consumo bajo de potencia que brinda un óptimo desempeño para el sistema de retroalimentación háptica, sus características se especifican en la Tabla 1.

Figura 2: Diseño del circuito impreso.

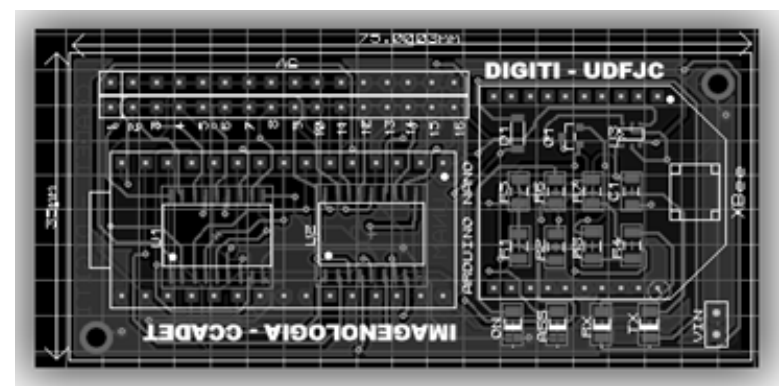

Fuente: elaboración propia.
El suministro de energía de la tarjeta electrónica debe ser suficiente para que cumpla con sus principales funciones de manera eficiente, por lo que se buscó un sistema de alimentación adecuado que diera energía para el funcionamiento continuo (varias horas o días) del sistema de control inalámbrico junto a los actuadores vibrotáctiles.

Tabla 1: Características del sistema de control inalámbrico.

\begin{tabular}{|c|l|}
\hline Característica & \multicolumn{1}{|c|}{ Descripción } \\
\hline Velocidad & \multicolumn{1}{|c|}{$8 \mathrm{MHz}$} \\
\hline FLASH & \multicolumn{1}{|c|}{$64 \mathrm{~Kb}$} \\
\hline Comunicación & $\begin{array}{l}\text { Conexión inalámbrica } \\
\text { XBee }\end{array}$ \\
\hline Consumo & $\begin{array}{l}50 \mathrm{~mA} \text { estado activo } \\
2 \mathrm{~mA} \text { estado inactivo }\end{array}$ \\
\hline Tamaño & $7.5 \mathrm{~cm} \mathrm{x} 3.5 \mathrm{~cm} \times 1 \mathrm{~cm}$ \\
\hline
\end{tabular}

Fuente: elaboración propia.

Luego de elegir el sistema de alimentación de potencia se acoplaron todos los sistemas y se hicieron pruebas de 
duración, en estas se obtuvieron resultados satisfactorios, activando los dieciséis actuadores al tiempo que se transmitían comandos de control inalámbricamente, logrando obtener una duración de más de dos días de uso continuo. El sistema es capaz de durar dieciséis veces más si se activa un actuador háptico a la vez, y al no trasmitir datos inalámbricamente de manera constante aumenta la eficiencia en potencia del dispositivo; se calcula que el dispositivo puede tener en promedio una autonomía de cuatro o cinco días.

El diseño industrial final que acopla todo el dispositivo consta de una caja aislante que contiene la tarjeta de desarrollo, un cilindro donde se encaja el sistema de alimentación y un brazalete para la sujeción de los dieciséis vibromotores y los puntos a estimular; estos actuadores se posicionaron de manera equidistante $(2 \mathrm{~cm})$ en dos columnas paralelas, cada una con ocho vibromotores para cubrir una distancia de $14 \mathrm{~cm}$ (Figura $3)$.

Figura 3: Diseño final del dispositivo portátil vibrotáctil. (a) Sistema electrónico de control; (b) interfaz final portátil montada en un individuo; (c) matriz de vibromotores del dispositivo.

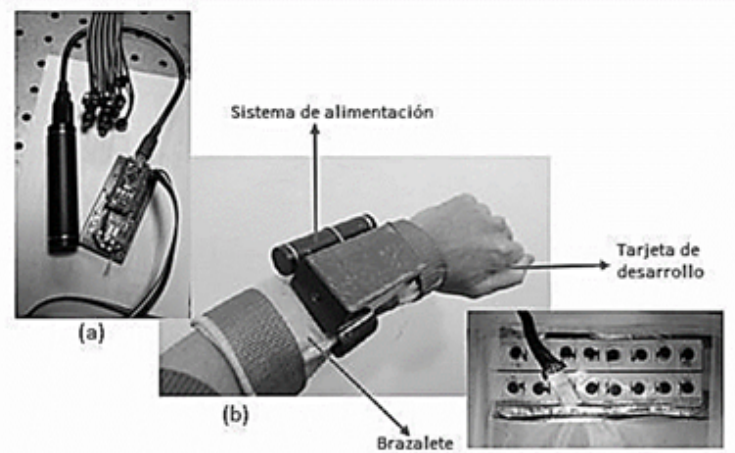

(c)

Fuente: elaboración propia.

Finalmente, para tener el control completo del dispositivo y poder interactuar con el sistema portátil se creó una interfaz gráfica de usuario que consta de dos partes, la primera es la parte de control que sirve para caracterizar los actuadores enviando comandos a través del módulo Xbee conectado al ordenador, también permitió generar sensaciones hápticas en diferentes puntos de la piel seleccionando los vibromotores y las frecuencias deseadas. La segunda parte la constituye una herramienta que permite experimentar el flujo vibrotáctil de una persona, generando sensaciones hápticas de forma secuencial y aleatoria con diferentes intensidades, lo cual puede ayudar a identificar la capacidad de discriminación entre diferentes estímulos en una persona.

\section{Pruebas de usabilidad}

Se realizaron tres tipos de experimentos con una persona sana sin discapacidad motriz ni déficit sensorial, lo anterior con fines particulares a la validación del flujo vibrotáctil que proporcionaba el dispositivo por medio de retroalimentaciones. El primer experimento consistió en evaluar el umbral de percepción táctil cutáneo con una serie de estímulos vibrotáctiles aplicados con intensidad variable; con este experimento se puede determinar la frecuencia umbral de percepción de las personas repitiendo tres veces el experimento para cada una y realizando la construcción de la gráfica que permitía evidenciar dicha frecuencia con variaciones en su voltaje. Con este método el dispositivo puede ser útil para evaluar la sensibilidad promedio de una persona sana, o bien para generar un mapa de sensibilidad por zonas en pacientes con problemas de sensibilidad táctil.

El segundo experimento consistió en establecer la discriminación entre dos estímulos diferentes, acotando la distancia en la que dos estímulos se activaban a la vez e identificando en qué momento se sentían como uno solo, lo cual podría servir como otra métrica para evaluar capacidad sensorial en pacientes. El tercer experimento consistió en evaluar la utilidad del instrumento para ajustar la capacidad de localización de estímulos vibrotáctiles variables que tiene una persona; este experimento permitió la validación del flujo vibrotáctil mediante la interacción con una imagen virtual y el dispositivo en cuestión (Figura 4), se debía localizar en un mapa de referencia el estímulo táctil, con y sin ayuda de una referencia visual.

\section{Resultados y discusión}

Para el caso del primer experimento, el participante fue capaz de detectar hasta una vibración mínima promedio de $30 \mathrm{~Hz}$ que corresponde a las frecuencias normales de detección de la piel. En el segundo experimento se encontró que cuando las frecuencias eran superiores a diez unidades (con respecto al nivel encontrado en el primer experimento) para los vibromotores que tenían una distancia de separación cercana a la distancia media se sentía solo un tipo de estímulo, por lo que se determinó que no era conveniente el uso de frecuencias superiores a esta; se encontró también que para sentir el estímulo en el punto en cuestión es necesario disminuir los niveles de intensidad que se le proporcionan al dispositivo para su funcionamiento, ya que en frecuencias muy altas y distancias muy cortas existe una percepción de saturación en la estimulación proporcionada. 
Figura 4: Experimento de localización de estímulos. (a) Imagen virtual de referencia visual del estímulo; (b) mapa de localización del estímulo en diferentes zonas el brazo.
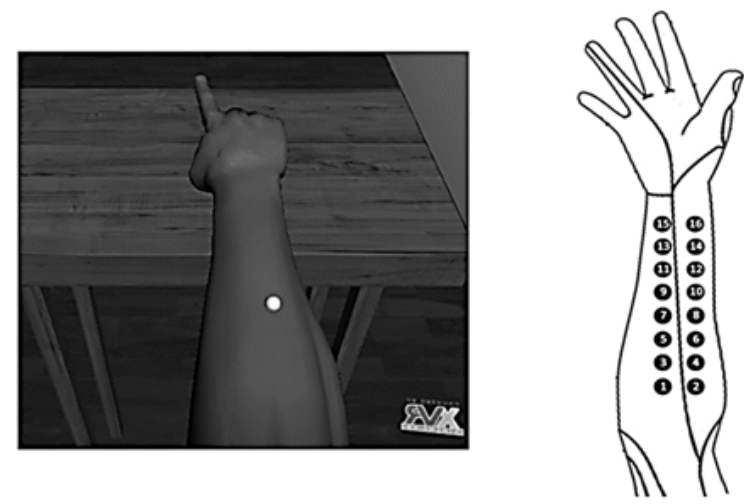

Fuente: elaboración propia.

Para el tercer experimento se encontraron resultados muy interesantes. Cuando se proporcionaban vibraciones en puntos cercanos y se señalaban otros punto de vibración en la imagen virtual, la persona señalaba tener una respuesta positiva (sensación de estimulación en el punto indicado) cuando no se realizaba la estimulación en este punto, sino en una zona cercana; esto muestra que en muchas de las ocasiones la persona puede ser influenciada psicológicamente para sentir estímulos donde realmente no se están estimulando, esto pasa sobre todo en personas que están presentando una deficiencia motriz en sus extremidades.

En este caso se obtiene que los errores de localización fueron menores cuando los participantes tuvieron la ayuda visual, el valor de error fue alrededor de un centímetro, que es la distancia de separación entre cada par de motores. Se piensa que sería bueno extender en el futuro esta prueba con más personas y probando mayores niveles de intensidad con el fin de investigar si esto se preserva o puede cambiar dependiendo de los niveles de vibración. Estas pruebas arrojaron como resultado el funcionamiento adecuado del sistema de retroalimentación, la validación del flujo vibrotáctil y una calibración final en los actuadores.

\section{Conclusiones}

En este trabajo se ha presentado el desarrollo de un dispositivo háptico portátil como método de biorretroalimentación en asistencia de actividades motoras como en la rehabilitación a pacientes con discapacidad motora transitoria y, de igual forma, para diagnosticar tempranamente a pacientes con neuropatías.

La validez del sistema ha sido justificada mediante experimentos controlados para evaluar el flujo vibrotáctil, tratando de discriminar o evaluar las posibles influencias de factores externos como el ruido de los vibromotores o el uso de la vista como distractor a la hora de recibir un estímulo táctil, para ello se realizaron tres experimentos con una persona sin discapacidad motriz o neuropatías. Al accionar los actuadores a altas frecuencias $i 120 \mathrm{~Hz}$ se pierde la capacidad de detectar un punto específico de vibración, ya que la fuerza vibratoria es muy alta y hace pensar que el elemento que lo genera es más grande que el área que ocupa en la piel, lo anterior lo hace ineficiente para validaciones del flujo vibrotáctil porque no permite la discriminación de dos o más estímulos cercanos, de igual manera mantenerlo mucho tiempo activo puede generar molestia o lesiones en la piel debido a la alta temperatura que toma el actuador con ese patrón de funcionamiento.

Finalmente, este dispositivo puede integrarse a aplicaciones de realidad virtual o videojuegos de entrenamiento para la rehabilitación de las extremidades superiores humanas. El dispositivo podría permitir estudiar la validez, la eficacia y eficiencia de este tipo de tecnologías en el ámbito sensorial háptico; además, podría tener posibles aplicaciones para la evaluación sensorial en las extremidades de otro tipo de pacientes con problemas de sensibilidad (por ejemplo, en diabéticos debido al desarrollo de neuropatías en sus extremidades). También se considera que este dispositivo podría ser valioso como herramienta de prevención, diagnóstico y de monitoreo en pacientes con otras neuropatías. Son estas las ventajas que tiene este dispositivo con respecto a otros, además de las características tecnológicas que lo hacen eficiente, moderno y adecuado.

\section{Reconocimientos}

Este trabajo se desarrolló con el fin de ser implementado en el proyecto de investigación "Estudio de la incidencia de la alineación estática en los parámetros cinemáticas de pacientes amputados transtibiales por causa de minas antipersonales", del grupo de investigación DIGITI institucionalizado por el CIDC de la Universidad Distrital Francisco José de Caldas. Este sistema se desarrolló e implementó en la unidad de investigación y desarrollo tecnológico del CCADET en el Hospital General de México "Dr. Eduardo Liceaga" de la Universidad Nacional Autónoma de México UNAM en la línea de rehabilitación asistida, quienes financiaron el desarrollo completo del proyecto. 


\section{Referencias}

[1] M. H. Beers, R. Berkow. "The Merck manual of diagnosis and therapy", New Jersey: Merck Research Laboratories, pp. 283-292, 1999.

[2] A. Frisoli, F. Salsedo, M. Bergamasco, B. Rossi, y M. C. Carboncini, "A force-feedback exoskeleton for upper-limb rehabilitation in virtual reality", Applied Bionics and Biomechanics, vol. 6, $\mathrm{n}^{\circ}$. 2, pp. 115-126, 2009. https : //doi.org/10.1155/2009/378254

[3] M. A. Padilla-Castaneda, E. Sotgiu, A. Frisoli, M. Bergamasco, P. Orsini, A. Martiradonna, S. Olivieri, G. Mazzinghi y C. Laddaga, "A virtual reality system for robotic-assisted orthopedic rehabilitation of forearm and elbow fractures", IEEE/RSJ International Conference on Intelligent Robots and Systems, 2013, pp. 1506-1511. https: //doi.org/10.1109/IROS.2013.6696548

[4] M. S. Cameirao, S. Bermúdez, E. Duarte, A. Frisoli y P. F. M. J. Verschure, "The combined impact of virtual reality neurorehabilitation and its interfaces on upper extremity functional recovery in patients with chronic stroke" Stroke, vol. 43, $\mathrm{n}^{\circ}$. 10, pp. 2720-2728, 2012. https://doi.org/10.1161/ STROKEAHA.112.653196

[5] A. Frisoli, C. Procopio, C. Chisari, I. Creatini, L. Bonfiglio, M. Beramasco, B. Rossi y M.C. Carboncini, "Positive effects of robotic exoskeleton training of upper limb reaching movements after stroke" Journal of Neuro Engineering and Rehabilitation, vol. 9, $\mathrm{n}^{\circ}$. 1, pp. 1-16, 2012. https : //doi .org/10.1186/1743-0003-9-36

[6] J. Chirivella, A. Barco, S. Blasco, V. Penades, G. Mas, y P. Gagliardo, "Neuro@ homeA software platform of clinically designed videogames designed for the cognitive rehabilitation of stroke patients", Brain Injury, 2014.

[7] A. L. Faria, A. Andrade, L. Soares, y S. Bermúdez, "Benefits of virtual reality based cognitive rehabilitation through simulated activities of daily living: a randomized controlled trial with stroke patients", Journal of NeuroEngineering and Rehabilitation, vol. 13, $\mathrm{n}^{\circ} . \quad 1,2016$. https : //doi.org/10.1186/s12984-016-0204-z

[8] F. J. Badesa, R. Morales, N. Garcia-Aracil, J. M. Sabater, A. Casals, y L. Zollo, "Autoadaptive robot-aided therapy using machine learning technique", Computer Methods and Programs in Biomedicine, vol. 116, $\mathrm{n}^{\circ}$. 2, pp. 123-130, 2014. https://doi.org/10.1016/j.cmpb.2013.09.011

[9] O. M. Giggins, U. M. Persson y B. Caulfield, "Biofeedback in rehabilitation", Journal of NeuroEngineering and Rehabilitation, vol. 10, $\mathrm{n}^{\circ}$. 1, 2013. https://doi.org/10.1186/ 1743-0003-10-60 